\title{
Field-Induced Quantum Critical Point in $\mathrm{CeCoIn}_{5}$
}

\author{
Johnpierre Paglione M. A. Tanatar \\ R. W. Hill, F. Ronning, M. Sutherland, and Louis Taillefen \\ Department of Physics, University of Toronto, Toronto, Ontario M5S 1A\%, Canada \\ C. Petrovid $\$$ and P. C. Canfield \\ Ames Laboratory and Department of Physics and Astronomy, Iowa State University, Ames, Iowa, 50011
}

(Dated: September 13, 2018)

\begin{abstract}
The resistivity of the heavy-fermion superconductor $\mathrm{CeCoIn}_{5}$ was measured as a function of temperature, down to $25 \mathrm{mK}$ and in magnetic fields of up to $16 \mathrm{~T}$ applied perpendicular to the basal plane. With increasing field, we observe a suppression of the non-Fermi liquid behavior, $\rho \sim T$, and the development of a Fermi liquid state, with its characteristic $\rho=\rho_{0}+A T^{2}$ dependence. The field dependence of the $T^{2}$ coefficient shows critical behavior with an exponent of 1.37. This is evidence for a field-induced quantum critical point (QCP), occuring at a critical field which coincides, within experimental accuracy, with the superconducting critical field $H_{c 2}$. We discuss the relation of this field-tuned QCP to a change in the magnetic state, seen as a change in magnetoresistance from positive to negative, at a crossover line that has a common border with the superconducting region below $\sim 1 \mathrm{~K}$.

PACS numbers: 71.27.+a,73.43.Qt,75.40.-s
\end{abstract}

The recent discovery of a new family of heavy-fermion superconductors with the general formula $\mathrm{CeMIn}_{5}$ ( $\mathrm{M}=\mathrm{Co}$, Ir, Rh or their solid solutions) has generated much interest. The ground state of these compounds can be fine-tuned between magnetic order, superconductivity, and a coexistence of the two by varying both pressure and alloy composition [1]. $\mathrm{CeCoIn}_{5}$, with the highest ambient pressure superconducting transition temperature $T_{c}$ of all heavy-fermion compounds discovered to date 2], is believed to be close to a point where the magnetic state becomes unstable as $T \rightarrow 0[3,4,[5]$. This proximity to a quantum critical point (QCP) is believed to be responsible for the unusual properties observed in the superconducting [6, 7, 8, 9, 10] and normal 11] states.

The peculiar magnetic properties of $\mathrm{CeCoIn}_{5}$ are determined by the magnetic moments of $\mathrm{Ce}^{3+}$ ions and by conduction electrons. Through systematic studies of $\mathrm{Ce}_{1-x} \mathrm{La}_{x} \mathrm{CoIn}_{5}$ alloys, where the dilution of magnetic Ce ions by La (non-magnetic analog) allows one to study the nature of intersite interactions, it has been shown that the energy scales associated with the relevant magnetic interactions are all well separated, and that the dominance of direct intersite interactions below the coherence peak temperature $T_{\text {coh }} \sim 50 \mathrm{~K}$ gives rise to pronounced two-dimensional antiferromagnetic (AF) correlations 12. Although long-range magnetic order is not present in $\mathrm{CeCoIn}_{5}$ [13], the close proximity of this system to AF order 7] results in an abundance of spin fluctuations which lead to behavior that is notably different from that expected in the Fermi liquid (FL) model.

In addition to the observation of $T$-linear resistivity [2] commonly associated with quantum criticality, nonFermi liquid (NFL) behavior manifests itself in $\mathrm{CeCoIn}_{5}$ in a number of ways. These include a logarithmic increase of the electronic specific heat coefficient on cool- ing 14, an enhancement of the effective mass at low temperatures and its strong field dependence, as seen in de Haas-van Alphen [15] and microwave conductivity [16] experiments, and a magnetic susceptibility that does not saturate at low temperatures [2].

The close proximity of $\mathrm{CeCoIn}_{5}$ to an $\mathrm{AF}$ QCP at ambient pressure offers a unique opportunity to explore quantum critical phenomena in a system free of disorder due to alloying. Since magnetic fluctuations play an essential role in quantum criticality, the response of this system to applied magnetic fields is of clear interest. We have performed a systematic study of the low temperature electrical resistivity of $\mathrm{CeCoIn}_{5}$ in magnetic fields up to $16 \mathrm{~T}$. We find that this system can indeed be driven through a QCP by a magnetic field, as evidenced by a divergence of the electron-electron scattering strength at the critical field and the subsequent restoration of a FL state. We show that magnetic interactions appear to play a key role in this critical behavior.

Single crystals of $\mathrm{CeCoIn}_{5}$ were grown by the selfflux method [2]. As-grown crystals have a thin platelet shape, with large surfaces corresponding to the (001) basal plane. For this study we have cut four samples into rectangular parallelepipeds with typical dimensions $\sim 2 \times 1 \times 0.3 \mathrm{~mm}$. Electrical contacts for standard fourwire measurements were made with soldered indium, resulting in $\sim 5 \mathrm{~m} \Omega$ contact resistances. The in-plane electrical resistivity, $\rho$, was measured with an $\mathrm{AC}$ resistance bridge by applying $0.1 \mathrm{~mA}$ excitation currents along the basal plane, and transverse magnetic fields up to $16 \mathrm{~T}$ parallel to the [001] axis. Measurements were performed from $300 \mathrm{~K}$ to $0.3 \mathrm{~K}$ in a ${ }^{3} \mathrm{He}$ cryostat, and down to $25 \mathrm{mK}$ in a ${ }^{3} \mathrm{He} /{ }^{4} \mathrm{He}$ dilution refrigerator. The $16 \mathrm{~T}$ data were reproduced in reversed field direction, excluding any spurious Hall component. 
On cooling from room temperature, the zero-field resistivity of $\mathrm{CeCoIn}_{5}$ shows a slight increase, followed by a crossover to metallic behavior below $T_{\text {coh }}$, as is seen in many heavy-fermion systems [17]. Below $\sim 10 \mathrm{~K}$, $\rho(T)$ displays a linear temperature dependence down to $T_{c}=2.3 \mathrm{~K}$, as shown in Fig. 17. Such $T$-linear behavior has been observed in many systems which lie at or close to a QCP [17, 18]. Above $30 \mathrm{~K}$, the magnetoresistance (MR) is negligibly small up to $16 \mathrm{~T}$, whereas below $30 \mathrm{~K}$ a notable MR begins to develop as shown in Fig. 1b. The linear dependence of $\rho(T)$ observed at $H=0$ suffers a drastic change with increasing field, most pronounced at low temperatures. For instance, the $6 \mathrm{~T}$ curve (Fig. 19) displays a notable deviation from linearity below $\sim 5 \mathrm{~K}$ and decreases on cooling to a value much lower than the residual resistivity inferred from a linear extrapolation of $\rho(T, H=0)$ to $T=0$. By $16 \mathrm{~T}$, the downturn in $\rho(T)$ shifts to much higher temperatures (Fig. 1h), as evidenced by the large negative MR below $\sim 5 \mathrm{~K}$ shown in Fig. 1b. At higher temperatures, the field dependence of $\rho$, plotted at constant $T$ values in Fig. 1 , reveals the development of a crossover in the sign of MR with increasing field and temperature, which will be discussed below.

A close analysis of our $6 \mathrm{~T}$ data at low temperatures reveals a narrow but clearly distinguishable range of $T^{2}$ behavior below $\sim 100 \mathrm{mK}$, highlighted in a plot of $\rho$ vs. $T^{2}$ in Fig. ??. This range gradually becomes wider and more apparent with increasing field, as shown by the linear fits [19] in the main panel of Fig. 2] and extends to as high as $2.5 \mathrm{~K}$ by $H=16 \mathrm{~T}$. Simultaneously, at the lowest measured temperatures, a small upturn in $\rho(T)$ starts to develop above $8 \mathrm{~T}$ and continues to grow upon further field increase. Since this effect is confined to very low temperatures and high fields, it does not hinder the observation of $T^{2}$ resistivity and is left for future study 21.

The behavior of $\mathrm{MR}$ in $\mathrm{CeCoIn}_{5}$ can be compared to that observed in two closely related superconductors. In $\mathrm{CeIrIn}_{5}$, no significant MR has been observed between $50 \mathrm{mK}$ and $5 \mathrm{~K}$ at ambient pressure [2]. In $\mathrm{CeRhIn}_{5}$, a large positive MR was observed [23] at ambient pressure, while under the critical pressure (where AF order is suppressed and superconductivity is dominant) the low temperature MR 24] bears a striking similarity to that observed in our study. The development of a $T^{2}$ dependence of resistivity was also seen in $\mathrm{CeCoIn}_{5}$ under applied pressure, where a jump in the exponent of $T$ from linear to quadratic occurs near 2 GPa [5]. From these comparisons, it would appear that the Co system at ambient pressure is close to quantum criticality in the same manner as the Rh system under critical pressure, while the Ir system is further from criticality at ambient pressure.

The slope of the fitted $\rho$ vs. $T^{2}$ curves (Fig [2), i.e. the coefficient $A$ of the $T^{2}$ term (in $\rho=\rho_{0}+A T^{2}$ ), is a measure of the strength of electron-electron interactions,
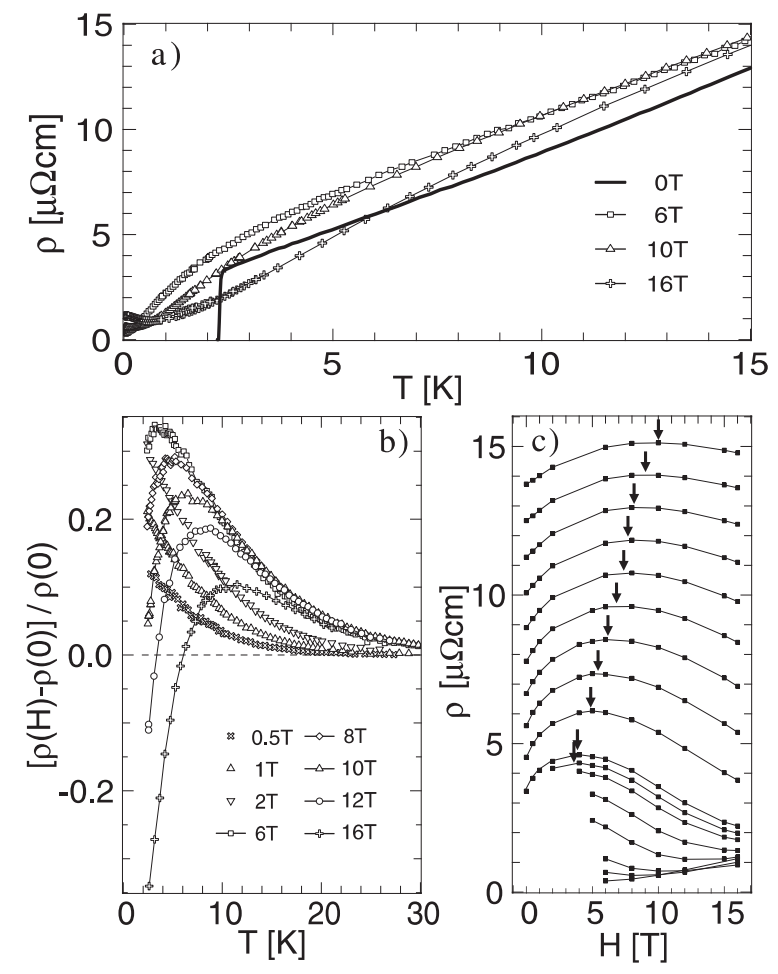

FIG. 1: Resistivity of $\mathrm{CeCoIn}_{5}$ under various fields, shown a) for $H=0,6$, and $16 \mathrm{~T}, \mathrm{~b}$ ) as magnetoresistance versus temperature, normalized to zero field resistivity, and c) versus applied field, as isotherms for $T=16,14.5,13,11.5,10$, $8.5,7,5.5,4,2.5,2.25,2,1.5,1,0.5,0.3$ and $0.1 \mathrm{~K}$ from top to bottom (lines are guides to the eye and are not offset). The arrows in c) indicate the position of the crossover from positive to negative MR with increasing field for each temperature.

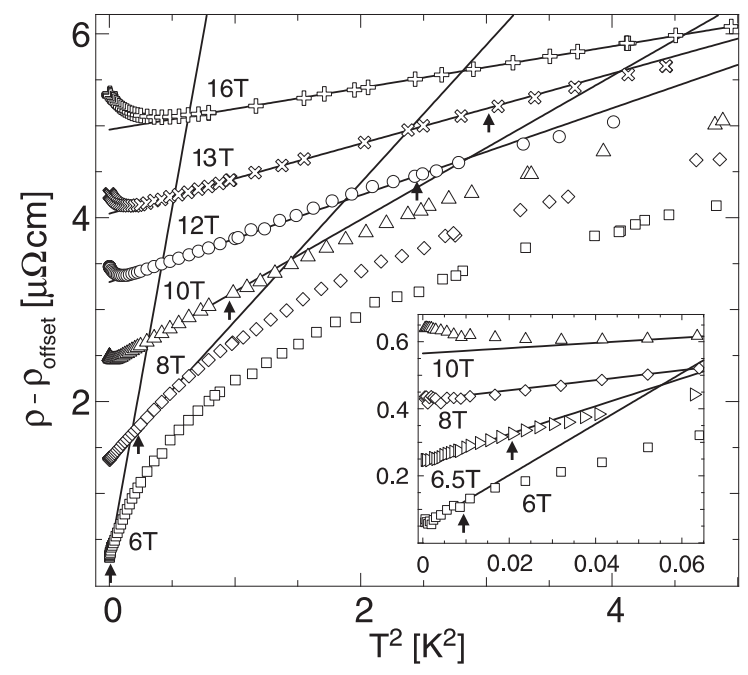

FIG. 2: Low temperature resistivity of $\mathrm{CeCoIn}_{5}$ plotted vs. $T^{2}$ for several magnetic fields, with an inset showing low temperature data (data sets are offset for clarity in both figures). The solid lines are linear fits to the data, showing the behavior of the quadratic-term coefficient (slope) under applied field, with arrows indicating the upper limit of the temperature range of $T^{2}$ behavior. 
notoriously high in heavy-fermion materials. As is clear from the fits in Fig. 2] $A$ tends to decrease with increasing field. The field dependence of $A$, or $A(H)$ (inset in Fig. (3), displays critical behavior best fitted by the function $A \propto\left(H-H^{*}\right)^{\alpha}$, with parameters $H^{*}=5.1 \pm 0.2 \mathrm{~T}$ and $\alpha=-1.37 \pm 0.1$. In FL theory, the coefficient $A$ is roughly proportional to the square of the electronic contribution to the specific heat coefficient $\gamma$. Thus, the critical behavior of $A(H)$ would lead us to expect a divergence of $\gamma(H)$ somewhere close to $H_{c 2}$. Experimental studies of the specific heat at fields slightly exceeding $H_{c 2}$ indeed show an increase of $\gamma(H)$ [14], but unfortunately detailed data is lacking in the field range of interest to allow a direct comparison.

The field-induced recovery of a FL regime in $\mathrm{CeCoIn}_{5}$ exhibits a distinct similarity to the behavior observed in several other systems. In $\mathrm{Sr}_{3} \mathrm{Ru}_{2} \mathrm{O}_{7}$ and $\mathrm{CeRu}_{2} \mathrm{Si}_{2}$, a field-induced anomaly in resistivity is associated in both cases with a change from predominantly AF to ferromagnetic fluctuations [25]. In $\mathrm{U}_{0.9} \mathrm{Th}_{0.1} \mathrm{Be}_{13}$ [20], a system close to a superconducting phase, the evolution of $A(H)$ and $T^{2}$ resistivity with field both bear a close resemblance to that found in our study. In $\mathrm{YbRh}_{2} \mathrm{Si}_{2}$, critical behavior in $A(H)$ was observed in proximity to a field-induced QCP associated with a second-order AF transition, with an exponent $\alpha=-1$ extracted from $A(H)$ that is similar to the value we obtain in $\mathrm{CeCoIn}_{5}$ [26].

All of the aforementioned systems exhibit critical behavior in resistivity when approaching some critical field value $H^{*}$. However, what is unique (and intriguing) about $\mathrm{CeCoIn}_{5}$ is the fact that $H^{*}$ is very close to $H_{c 2}(0)$, which points to the existence of a QCP coincident with the superconducting transition at $T=0$. The question is whether this coincidence is essential or accidental. In the latter case, the critical behavior would originate from proximity to an ordered phase (and transition) other than the superconducting state (and in fact would be masked by superconductivity). In this respect it is interesting to mention the compelling evidence from magnetization [27, 28], specific heat 29], and thermal conductivity [9] that the $H_{c 2}$ transition in $\mathrm{CeCoIn}_{5}$ is first-order below $\sim 0.7 \mathrm{~K}$ for all field orientations. This suggests that critical behavior of the kind observed here, which is usually associated with a second-order phase transition brought to absolute zero, is not caused by the vicinity of the superconducting state itself. Rather, it is tempting to propose that the quantum critical behavior observed in $\mathrm{CeCoIn}_{5}$ is associated with a zero-temperature transition of magnetic origin, much as the field-induced transition from an AF state to a field-aligned state in $\mathrm{YbRh}_{2} \mathrm{Si}_{2}$.

In connection to this, note that there is a pronounced crossover from positive to negative MR that occurs at high temperatures with increasing field. It is clear that the shape of the $\rho(H)$ curves is notably different from that expected for weak-field orbital MR $\left(\Delta \rho \sim H^{2}\right)$, while at high fields the MR becomes negative. Both of

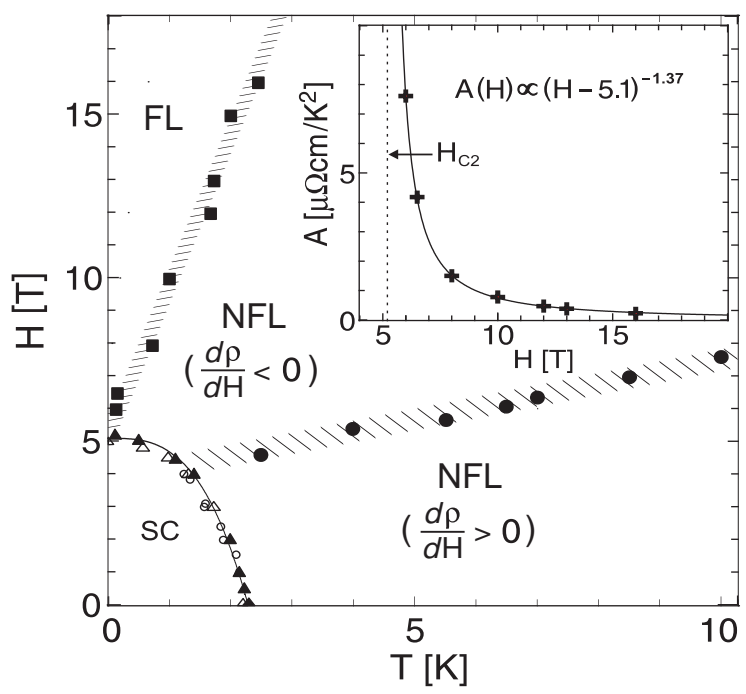

FIG. 3: $H-T$ phase diagram of $\mathrm{CeCoIn}_{5}$ determined from resistivity measurements, including the upper bound of $T^{2}$ resistivity $(\boldsymbol{\square})$, the position of the $\operatorname{MR}$ maximum $(\bullet)$, and $H_{c 2}$ determined by resistivity $H$ - and $T$-sweeps $(\boldsymbol{\Lambda})$, which agrees with specific heat $(\circ)$ and resistivity $(\triangle)$ measurements from Ref. 2. The hatched lines indicate the crossover boundaries between FL and NFL (negative and positive MR) regimes, as explained in the text. The inset shows the field dependence of the quadratic coefficient $A$ of $\rho(T)$ (solid line is a fit of the data points $(+)$ to the displayed formula).

these facts encourage us to consider an unconventional magnetic origin to the observed MR behavior. This conclusion is natural, taking into account the connection commonly made between zero-field $T$-linear resistivity and AF spin fluctuations [17, 30]. In this scenario, the initial increase of $\rho$ with field could originate from an increase of spin disorder. Although positive MR is unexpected in Kondo systems [31], an increase of MR with field is indeed observed in systems with AF order 23, 26], and in systems approaching a coherent state [32]. In $\mathrm{CeCoIn}_{5}$, there is no evidence for long-range $\mathrm{AF}$ order in zero external field. However, notable AF correlations are observed below $T_{c o h}$, and therefore it is natural to associate the increase of spin disorder with a suppression of AF correlations. Clearly, the polarization of spins by increasing field strength should eventually lead to a fieldaligned state, as was observed in the case of $\mathrm{YbRh}_{2} \mathrm{Si}_{2}$ [26]. Therefore, a crossover to negative MR should occur at progressively increasing fields at higher temperatures, which is indeed observed in our experiment.

Based on our experimentally determined ranges of $T^{2}$ behavior at low temperatures, and on the crossover observed in the sign of MR at high temperatures, we have constructed a phase diagram of the $H-T$ plane (Fig 3 . It is apparent that the MR crossover line approaches the $H_{c 2}$ transition at a finite temperature, so that below $\sim 1 \mathrm{~K}$ the domain of negative MR is directly adjacent to the superconducting domain. Surprisingly, this 
diagram strongly resembles that determined by torque magnetometry [27] for $H \|$ [110], where the jump in torque associated with the first-order $H_{c 2}$ transition below $\sim 1.4 \mathrm{~K}$ was traced well into the normal state (up to $\sim 25 \mathrm{~K}$ ), indicative of a metamagnetic transformation. Although no such anomaly was found above $T_{c}$ for $H \|$ [100] or $H \|$ [001], and subsequent magnetization measurements did not reproduce this result [28], the sensitivity of torque measurements may highlight the importance of the $H \|$ [110] orientation. The similar behavior observed in both MR and torque measurements further suggests the possible existence of a magnetic order parameter, where the direct observation of a transition may be complicated by the emergence of superconductivity.

In conclusion, we have identified the anomalous lowtemperature evolution of magnetoresistance in $\mathrm{CeCoIn}_{5}$ with the field-induced development of a Fermi liquid regime. This evolution and the critical nature of the electron-electron scattering coefficient thus derived both bear close resemblance to other systems governed by quantum criticality. The crossover from positive to negative magnetoresistance, which extends to high temperatures, is indicative of a change in character of spin fluctuations with increasing field strength, and may be closely tied to the critical behavior observed in the resistivity, hinting at a quantum critical point of a magnetic nature, masked by superconductivity.

This work was supported by the Canadian Institute for Advanced Research and funded by NSERC. The authors gratefully acknowledge useful discussions with J. Hopkinson, Y. B. Kim, J. L. Sarrao, and I. Vekhter.

Note added.- Recent specific heat measurements by Bianchi et al. 33] support our main conclusion regarding the existence of a field-induced QCP.

* Electronic address: paglione@physics.utoronto.ca

$\dagger$ Permanent address: Inst. Surface Chemistry, N.A.S. Ukraine, Kyiv, Ukraine.

$\ddagger$ Current address: Département de physique, Université de Sherbrooke, Sherbrooke, Canada, J1K 2R1.

$\S$ Current address: Department of Physics, Brookhaven National Laboratory, Upton, New York, 11973.

[1] P.G. Pagliuso et al., Physica B 312, 129 (2002); P.G. Pagliuso et al., Phys. Rev. B 64, 100503R (2001); V. S. Zapf et al., Phys. Rev. B 65, 014506 (2001).

[2] C. Petrovic et al., J. Phys. Condens. Matter 13, L337 (2001).

[3] M. Nicklas et al., J. Phys. Condens. Matter 13, L905 (2001).

[4] H. Shishido et al., J. Phys. Soc. Jpn 71, 162 (2002).

[5] V.A. Sidorov et al., Phys. Rev. Lett. 89, 157004 (2002).

[6] R. Movshovich et al., Phys. Rev. Lett. 86, 5152 (2001).

[7] Y. Kohori et al., Phys. Rev. B 64, 134526 (2001).

[8] N. J. Curro et al., Phys. Rev. B 64, 180514 (2001).

[9] K. Izawa et al., Phys. Rev. Lett. 87, 057002 (2001).
[10] R. J. Ormeno et al., Phys. Rev. Lett 88, 047005 (2002).

[11] J.L. Sarrao, Int. J. Mod. Phys. B 16, 3025(2002).

[12] S. Nakatsuji et al., Phys. Rev. Lett. 89, 106402 (2002), and references cited therein.

[13] W. Higemoto et al., J. Phys. Soc. Jpn. 71, 1023 (2002).

[14] S. Ikeda et al. J. Phys. Soc. Jpn. 70, 3187 (2001); J. S. Kim et al., Phys. Rev. B 64, 134524 (2001).

[15] R. Settai et al., J. Phys. Soc. Jpn. 71, 162 (2002).

[16] S. Özcan et al., Europhys. Lett. 62, 412 (2003).

[17] See, for example, G. R. Stewart, Rev. Mod. Phys. 56, 755 (1984); G. R. Stewart, Rev. Mod. Phys. 73, 797 (2001).

[18] For a review, see P. Coleman et al., J. Phys. Condens. Matter 13, R7235 (2001); N. D. Mathur et al., Nature 394, 39 (1998).

[19] The range of $T^{2}$ behavior was determined using a procedure identical to that used in Ref. [20]. Linear leastsquares fits of the data were made over a varying interval of temperatures, fixing the lower bound at the lowest temperature point and varying the upper temperature bound. The reduced chi-square value was determined for each fit interval and plotted as a function of the upper bound of the fitting range. The upper bound corresponding to the minimum chi-square value was then chosen for the $T^{2}$ range.

[20] R. P. Dickey et al., Phys. Rev. B 56, 11169 (1997).

[21] This anomaly is similar to the upturn observed in the resistivity of $\mathrm{UPt}_{3}$ in transverse fields, where such a deviation from $T^{2}$ behavior at low temperatures is attributed to the fact that the $\omega_{c} \tau>1$ limit is realized [22]. In this limit, the characteristic time of the quasiparticle orbital motion is short compared with the time between collisions, and therefore the Fermi surface topology plays an important role in determining transport properties. Note that, due to the presence of this upturn, it was necessary to exclude the lowest temperature data from the $T^{2}$ fits (for $H>8 \mathrm{~T}$ ). This was accomplished by using the same chi-square minimization routine as described previously, but by also varying the lower temperature bound.

[22] L. Taillefer, F. Piquemal, and J. Flouquet, Physica C 153, 451 (1988); L. Taillefer, J. Flouquet, and W. Joss, J. Mag. Mag. Mat. 76, 218 (1988).

[23] A. D. Christianson et al., Phys. Rev. B 66, 054410 (2002).

[24] T. Muramatsu et al., Physica B 312, 140 (2002).

[25] J. Flouquet et al., Physica B 319, 251 (2002).

[26] P. Gegenwart et al., Phys. Rev. Lett. 89, 056402 (2002); J. Custers et al., Nature (London) 424, 524 (2003).

[27] T. P. Murphy et al., Phys. Rev. B 65, 100514(R) (2002).

[28] T. Tayama et al., Phys. Rev. B 65, 180504R (2002).

[29] A. Bianchi et al., Phys. Rev. Lett. 89, 137002 (2002).

[30] T. Moriya and K. Ueda, Adv. Phys. 49, 555 (2000); A. Rosch, Phys. Rev. B 62, 4945 (2000).

[31] M. D. Daybell and W. A. Steyert, Phys. Rev. Lett. 18, 398 (1967); E. T. Hedgcock et al., ibid. 20, 457 (1968); H. Rohrer, Phys. Rev. 174, 583 (1968); P. Monod, Phys. Rev. Lett. 19, 1113 (1967); R. More and H. Suhl, ibid. 20, 500 (1968); M. T. Béal-Monod and R. A. Weiner, Phys. Rev. 170, 552 (1968).

[32] U. Rauchschwalbe, F. Steglich, and H. Rietschel, Physica B 148, 33 (1987).

[33] A. Bianchi et al., Phys. Rev. Lett. (to be published). 